\title{
Single-shot optical conductivity measurement of dense aluminum plasmas
}

\author{
I. V. Churina, B.-I. Cho, A. Bernstein, D. S. Stoker, A. Dalton, D. R. Symes, and T. Ditmire \\ Department of Physics, Texas Center for High Intensity Laser Science, The University of Texas at Austin, Austin, Texas 78712, USA
}

(Received 16 October 2008; revised manuscript received 16 June 2009; published 9 July 2009)

\begin{abstract}
The optical conductivity of a dense femtosecond laser-heated aluminum plasma heated to $0.1-1.5 \mathrm{eV}$ was measured using frequency-domain interferometry with chirped pulses, permitting simultaneous observation of optical probe reflectivity and probe pulse phase shift. Coupled with published models of bound-electron contributions to the conductivity, these two independent experimental data yielded a direct measurement of both real and imaginary components of the plasma conductivity.
\end{abstract}

DOI: 10.1103/PhysRevE.80.015401

PACS number(s): 52.27.Gr, 52.25.Mq, 52.50.Jm

Intense femtosecond lasers offer a unique way to create and probe dense plasmas. In particular, femtosecond laserheated solid density plasmas can be used to examine the fundamental properties of strongly coupled strongly degenerate plasmas, such as electron thermal and electrical conductivity, particle transport, and energy deposition or the equation of state. There have been a number of previous studies on electron thermal transport and electrical conductivity of such ultrafast created plasmas with much of the information derived through optical reflectivity measurements [1-4]. The authors of these experiments have noted the difficulty of extracting the conductivity from reflectivity measurements off the target front surface when plasma density gradients form at this front surface [1,2]. Widmann [3] and Ping [4] presented successful measurements of conductivity in femtosecond laser-heated metals in which a thin foil with thickness of only a few skin depths was nearly uniformly heated by the femtosecond pulse. The ac conductivity was measured in these experiments by simultaneous probing of the foil's reflectivity and transmission on a time scale faster than hydrodynamic expansion, with these two quantities yielding the real and imaginary parts of the dielectric function. In these kinds of experiments, it is well known that as the intense femtosecond pulse enters the solid target foil, most of the energy is absorbed by the electrons, resulting in much lower ion temperature, a condition which lingers for a time up to the 1-2 ps time scale of electron-ion equilibration. One drawback to this technique is that it has some uncertainties in the density of such thin $(\sim 20-30 \mathrm{~nm})$ foils needed for the transmission measurement.

An alternate approach is to probe the back surface of a thicker metallic foil (which has little uncertainty in density) heated by a thermal conduction wave from the laser-heated front surface [5]. Such an approach requires measurement of both reflectivity and reflected probe phase shift to derive information on real and imaginary parts of the dielectric function. In this Rapid Communication we report on such a measurement, in which aluminum foils are heated by an intense, 35 fs laser pulse, and the dielectric function over a range of temperatures from $0.1-1.5 \mathrm{eV}$ are derived in a single shot as the temperature on the back surface ramps up from the arriving thermal wave. Using the technique of frequencydomain interferometry (FDI) [6] we simultaneously measure reflectivity and reflected pulse phase shift with subpicosecond time resolution. Accounting for the contribution of bound electrons to the optical conductivity, we compare our measurements of the conductivity with the calculated values using the well-known Lee and Moore model [7]. We find that the Lee and More model is accurate at temperature $>0.5 \mathrm{eV}$ but that it slightly overestimates the imaginary component of the conductivity at lower temperature.

Our experiment was performed on the THOR laser in the University of Texas at Austin. The laser is capable of delivering $35 \mathrm{fs}$ pulses at $800 \mathrm{~nm}$ with energy of $0.8 \mathrm{~J}$. The schematic of the experimental setup is shown in Fig. 1. We placed Al foils with thickness between 170 and $375 \mathrm{~nm}$ at the heating beam focus. These thicknesses were chosen so that the thermal wave created by the heating laser propagated ahead of the shock wave as described below. These targets were made by depositing aluminum vapor onto a $25 \times 25 \mathrm{~mm}$ silicon wafers. The silicon wafer target had a grid pattern that was etched away to make an array of freestanding $300 \mu \mathrm{m} \times 300 \mu \mathrm{m}$ aluminum foil squares. Because the probed surface was formed by vapor deposition on single crystal $\mathrm{Si}$ which was removed by subsequent etching, the Al surface was of very high optical quality. As with any experiment on $\mathrm{Al}$, an inevitable oxide layer does form on the target surface. The target was contained in a vacuum chamber, where an $800 \mathrm{~nm}, 35 \mathrm{fs}$ pulse was frequency doubled to produce a $\sim 7 \mathrm{~mJ}$ pulse at $400 \mathrm{~nm}$ for a heating pulse. Using the second harmonic of the fundamental pulse allowed us to improve the prepulse contrast ratio to and estimated level of $\sim 10^{-8}$. The $400 \mathrm{~nm}$ pulse was focused to a spot size of roughly $150 \mu \mathrm{m}$ (ensuring that all dynamics in the sub- $\mu \mathrm{m}$-thick foil were completely one dimensional) yielding an intensity of up to $5 \times 10^{14} \mathrm{~W} / \mathrm{cm}^{2}$. Any hot electron production from vacuum heating [8] or resonance absorption was eliminated by directing the beam near normal to the foil surface.

A portion of the $800 \mathrm{~nm}$ uncompressed beam was split from the main pulse and compressed to a chirped 28 ps pulse by a pair of gratings in a separate compressor. Time with the pump was confirmed by third-harmonic generation in a crystal placed at the chamber center. Two Michelson interferometers were used to produce two pairs of probe pulses, one pair interrogating the target and the other as a reference pair. The first interferometer contained a quarter-wave plate to split the pulses into two orthogonally polarized pulses with a 120 ps delay between them. The second interferometer split each of the orthogonally polarized pulses into two pulses, with one pulse reflecting from the back surface of the target and the other reflecting from the mirror. The 3 ps delay in- 


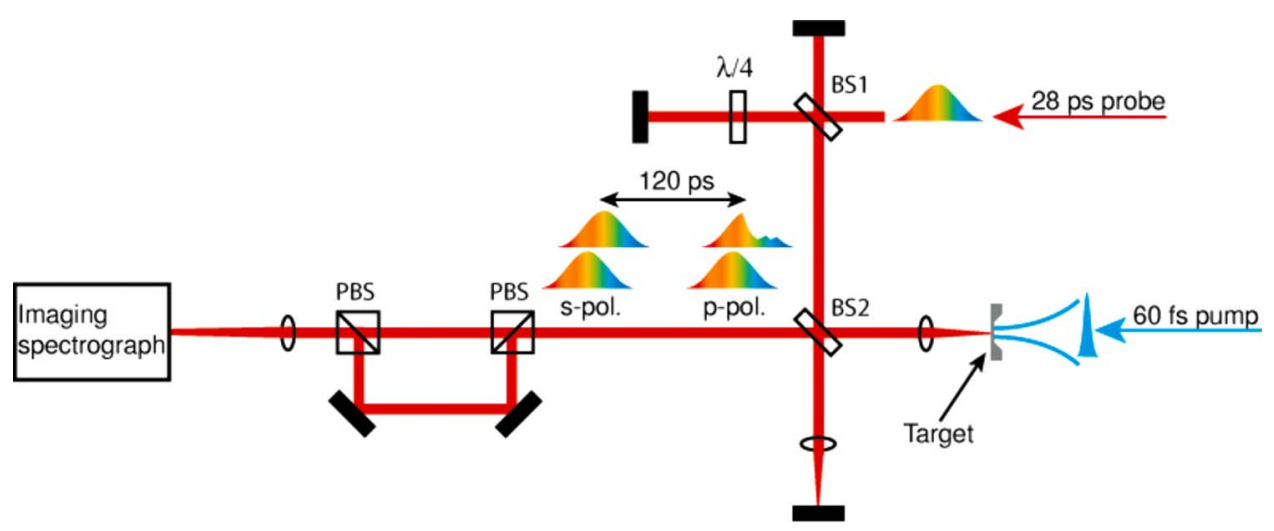

FIG. 1. (Color online) Schematic of the experimental setup. A $400 \mathrm{~nm}, 60 \mathrm{fs}$ pulse heats the front of the target. A 28 ps probe pulse was separated into two orthogonally polarized pulses by the first beam splitter (BS1). A second beam splitter (BS2) split each of the orthogonally polarized pulses into two pulses, with one of them reflecting from the back surface of the target. The resulting two sets of pulses interfere in the spectrometer and produce two interference patterns that were vertically separated using a pair of PBSs.

troduced between the split pulses in the second interferometer allowed us to produce spectral fringes resolvable by our spectrometer. The use of two orthogonally polarized pairs of pulses made it possible to separate spatially the pairs with two polarized beam splitters (PBSs) before entering the spectrometer. The heating beam was temporally overlapped with the second pair of pulses. The interference pattern produced by the first pair of pulses in the focal plane of the spectrometer was used to normalize the second interference pattern produced by the second pair of pulses. This allowed us to measure both the changes in the reflectivity and the phase shift of the probe pulse reflected from the back surface of the heated foil in a single shot. The spectral phase and reflectivity were extracted using a fast-Fourier-transform procedure [9] and converted to temporal phase and reflectivity using direct frequency-to-time mapping of the chirped pulse [10]. Following extraction, the optical parameters were temporally averaged to eliminate noise not removed by filtering, yielding a final temporal resolution of $350 \mathrm{fs}$.

Figure 2 shows the measured changes in phase and reflectivity of the optical probe reflected from the back surface of the heated $170 \mathrm{~nm}$ aluminum foil for various intensities. As seen from these graphs both quantities stayed the same for the first few ps and started to fall 5-6 ps after the heating pulse as the thermal wave from the front arrived at this back surface. As the temperature started increasing from room temperature, the conductivity decreased causing the reflectivity to drop and a greater skin depth for the probe. This changed the optical path and caused the reflected probe pulse's phase to change. When the shock front driven by the heating pulse reaches the back surface, the back side releases and the phase shift is subsequently dominated by particle motion as material expands. This appears as an increase in the rate of phase change drop at around 9 ps in the lower intensity data (triangles in Fig. 2). To demonstrate the final stages of this development, a line was fitted to the phase data at late time. The slope of the line is proportional to the free surface velocity, which is twice the particle velocity. The extracted particle velocity for the $170 \mathrm{~nm}$ foil was higher than the particle velocity extracted in earlier reports [11] of an aluminum film irradiated at the same intensity but is con- sistent with the smaller thickness of target and the fact that the shock decays as the pressure wave propagates. The fitted line shows a clear distinction between the stage when changes are dominated by the particle motion and the stage when the changes are defined by the temperature increase due to electron thermal conduction at early time. For several
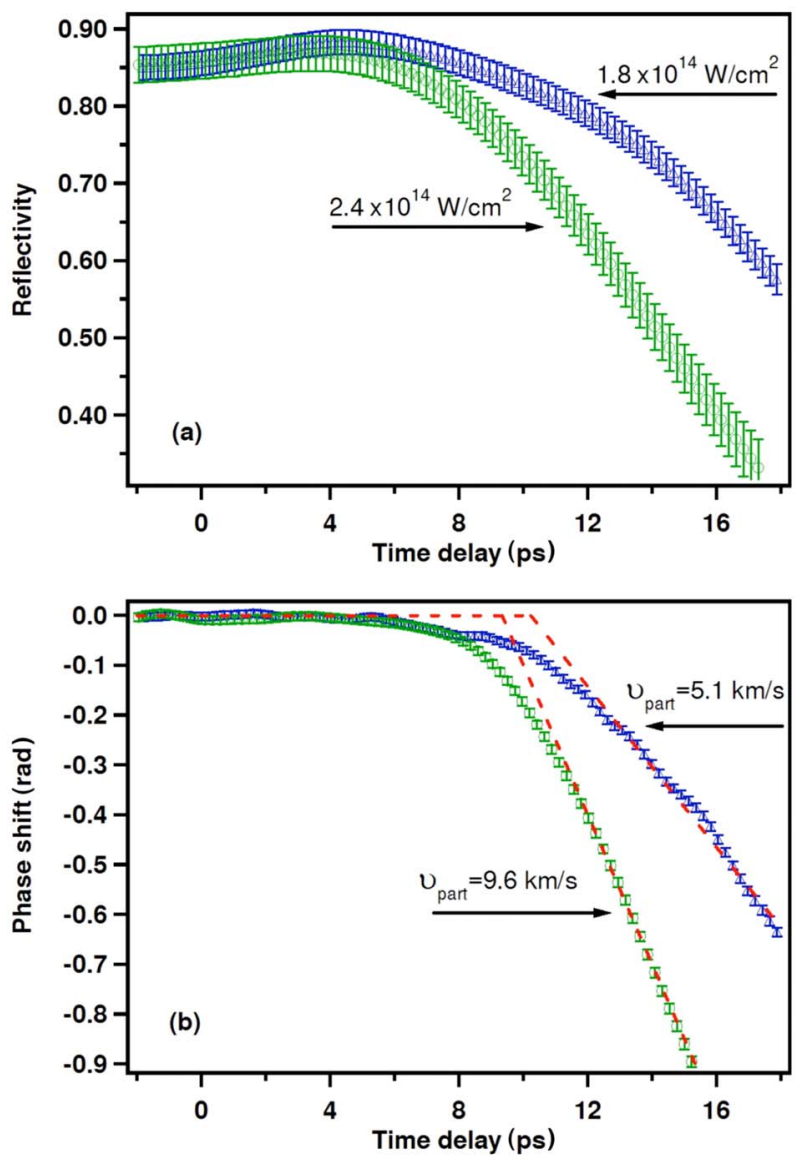

FIG. 2. (Color online) Time dependences of the back surface (a) reflectivity and (b) phase shift from 170-nm-thick laser-heated aluminum targets at $2.4 \times 10^{14} \mathrm{~W} / \mathrm{cm}^{2}$ (circles) and $1.8 \times 10^{14} \mathrm{~W} / \mathrm{cm}^{2}$ (triangles) intensities. 
ps prior to the shock breakout, the temperature on the back surface ramps up from the arriving thermal wave, while the heated back surface keeps a sharp interface to vacuum. During this time window, the reflectivity and phase changes allowed us to gain information about the heated solid.

With heating beam intensity of $(1-5) \times 10^{14} \mathrm{~W} / \mathrm{cm}^{2}$ the electron temperature within a skin depth immediately after laser absorption is $\sim 10-20 \mathrm{eV}$. Electron thermal conduction transfers heat through the rest of the solid density target, causing the temperature to rise in the foil. The temperature at the back of the target is 1-2 orders of magnitude smaller, resulting in temperatures below the Fermi temperature. The plasma created at the back surface is near solid density, is highly degenerate, and is strongly coupled.

To simulate the heat transfer in the heated foil the onedimensional hydrodynamic code HYADES [12] was used. To take into account the degeneracy and strongly coupled plasma effects, the Lee and More conductivity model [7] for dense plasmas was implemented in the code and the results compared to our measurements. To determine the foil optical response given the parameters yielded by HYADES, Maxwell's equations were solved via a standard matrix transfer method [13]. The dielectric function was given as $\varepsilon(x, \omega)=1+i 4 \pi \sigma(x, \omega) / \omega$, where $\omega$ is the laser frequency, $x$ is a coordinate into the back side of the target, and $\sigma(x, \omega)$ is the total optical conductivity. We assumed that the total optical conductivity at $800 \mathrm{~nm}$ contained contributions from both bound and free electrons such that $\sigma(x, \omega)=\sigma_{\text {free }}(x, \omega)+\sigma_{\text {bound }}(x, \omega)$. The free-electron contribution was described by a Drude model $\sigma_{\text {free }}(x, \omega)$ $=\sigma_{d c}(x) \nu(x) /[\nu(x)-i \omega]$, with a dc conductivity of $\sigma_{d c}(x)$, and an electron collision frequency $\nu(x)$. The bound (covalent) electron contribution was important because of the strong interband transition in aluminum at $1.5 \mathrm{eV}$ that is a result of parallel band structure in the planes parallel to the (200) faces of the Brillouin zone in the fcc aluminum [14].

While lattice properties do not change significantly before the pressure wave's arrival at the back surface, the increase in electron temperature causes an increase in collision frequency that changes the optical conductivity by shifting the interband absorption. The formulas for the real and imaginary parts of $\sigma_{\text {bound }}(x, \omega)$ are derived in Ref. [14]. In fact $\operatorname{Re}\left[\sigma_{\text {bound }}(x, \omega)\right]$ is significantly larger than $\operatorname{Re}\left[\sigma_{\text {free }}(x, \omega)\right]$ [15] indicating that the interband contribution is the dominant light absorption mechanism at $800 \mathrm{~nm}$ for roomtemperature aluminum. Both free and bound-electron contributions to the total optical conductivity are functions of collision frequency. We assume that the collision frequency in the studied temperature range was defined by the bulk properties of a foil $\nu(x)=\omega_{p l}^{2}(x) /\left[4 \pi \sigma_{d c}(x)\right]$, where $\omega_{p l}$ is the plasma frequency.

The output of the HYADES simulation $\sigma(x, \omega)$ was computed and incorporated in a Maxwell wave solver for calculating the optical properties of the probe at the foil surface. Figure 3 shows the results of a comparison between the calculated time-dependent reflectivity and phase shift using the Lee and More conductivity model and the experimental data obtained from a $170 \mathrm{~nm}$ aluminum foil irradiated at 2.4 $\times 10^{14} \mathrm{~W} / \mathrm{cm}^{2}$. The shock breakout time of $8.5 \mathrm{ps}$ obtained from the hydrodynamic simulation reproduces the experi-
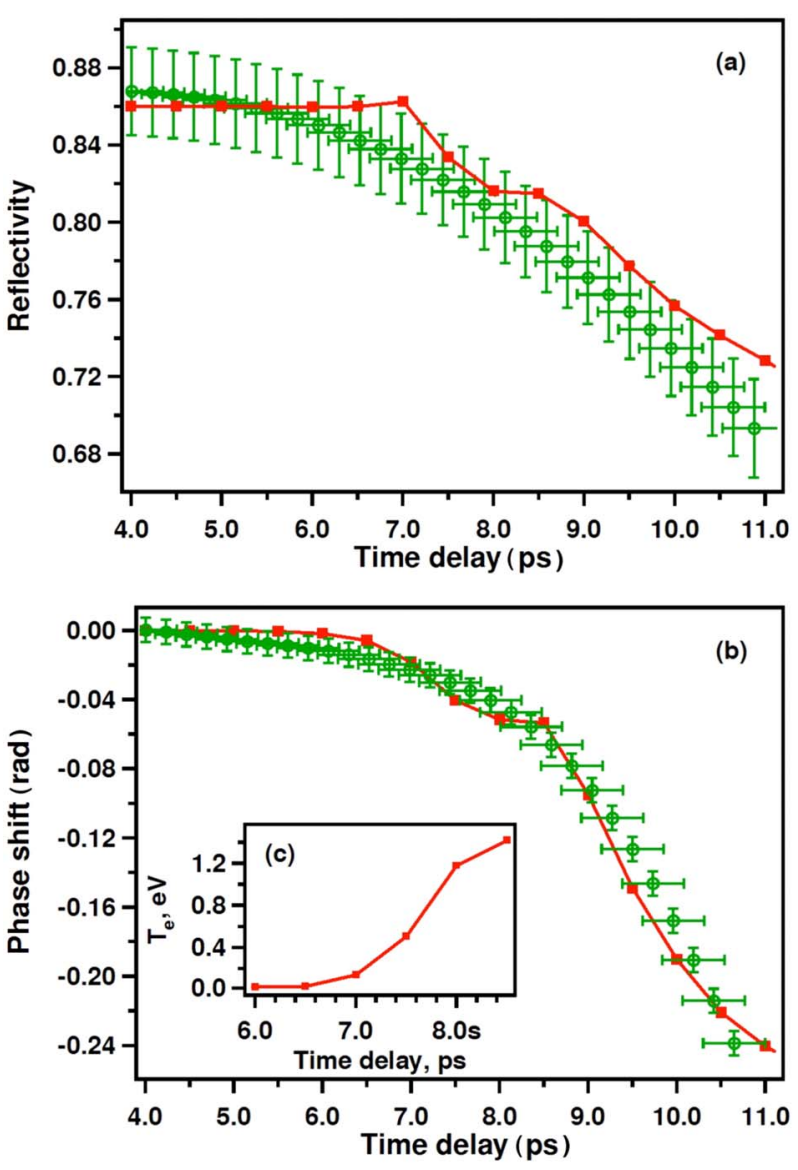

FIG. 3. (Color online) Time dependences of the (a) reflectivity and (b) phase shift changes from the laser-heated $170 \mathrm{~nm}$ aluminum target at $2.4 \times 10^{14} \mathrm{~W} / \mathrm{cm}^{2}$ (circles) compared to the calculated ones (squares) obtained using HYADES output. (c) Time dependence of the back surface average electron temperature obtained in HYADES.

mental results. It is informative, then to look at the dynamics of the foil in the first $8.5 \mathrm{ps}$, corresponding to the time before the shock wave's arrival at the back surface. The electron temperature changes at the back surface prior to the shock breakout, as shown in Fig. 3(c). For 3 ps the back surface of the target sees a continuous transition from room temperature to few $\mathrm{eV}$, while the heated back surface keeps a sharp interface to vacuum (well under one wavelength). As is shown in Ref. [16] a reflectivity decrease is correlated with a temperature increase and not with the increase in internal target pressure. In the time window before the pressure wave (shock wave) reaches the back surface of the target, the plasma is at solid density.

Using these dynamics we were able to extract, on a single shot, the dielectric constant, and optical conductivity simultaneously over the $0.1-1.5 \mathrm{eV}$ temperature range using Fresnel's equations. We have found that the shot to shot reproducibility is excellent. In Fig. 4 the extracted real and imaginary parts of the optical conductivity as a function of temperature are compared to calculated values using the Lee and More conductivity model. (Error bars in this plot represent both statistical and systematic error.) As the temperature increases, the imaginary part of the optical conductivity 

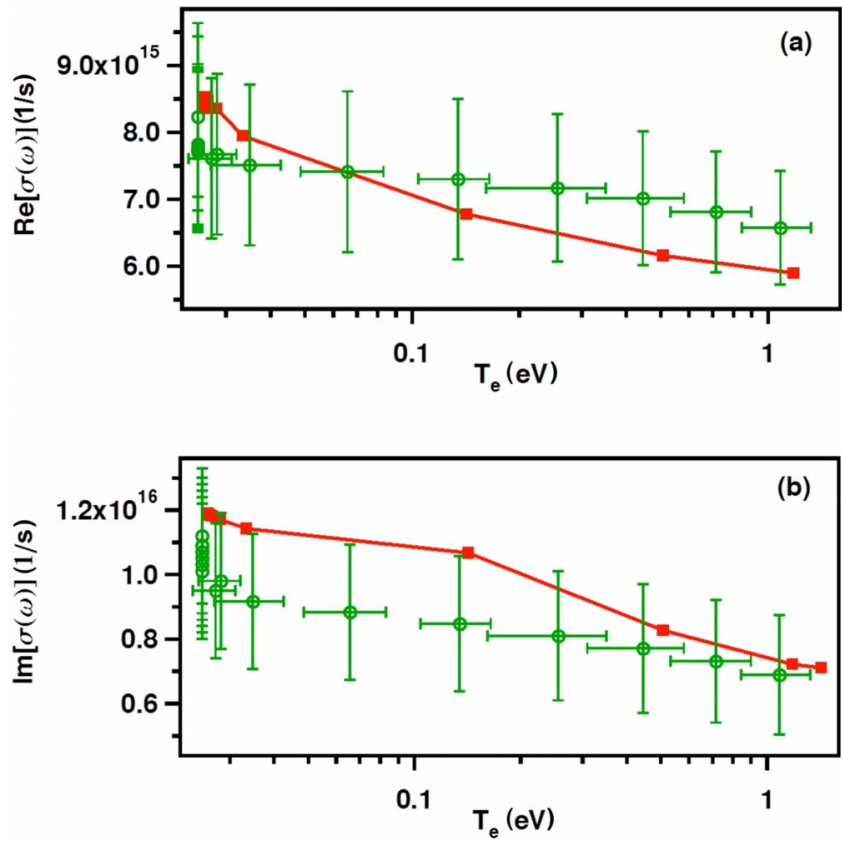

FIG. 4. (Color online) Temperature dependences of (a) real and (b) imaginary parts of optical conductivity extracted from the measured optical parameters (circles) compared to the calculated ones (squares) obtain using HYADES output.

drops, indicative of the Ohmic-like conductivity of a metal. The real part shows more complicated temperature dependence; it approaches a stable value after the temperature has risen well above room temperature. There is, however, some difference between the measured real and imaginary conductivities and that of the Lee and More model particularly at temperature below $0.1 \mathrm{eV}$. At our probe frequency the dominant absorption mechanism is the interband absorption, indicating that the discrepancy is caused mostly by the boundelectron contribution to the conductivity though temperature- dependent changes in the lattice are not taken into account [17]. We note that in some previous work it has been shown that the calculated Lee and More dc conductivity is at least an order of magnitude higher than measured values for warm $(<0.6 \mathrm{eV})$ moderately dense $\left(1.6 \mathrm{~g} / \mathrm{cm}^{3}\right)$ aluminum [18]. Our data at solid density $\left(2.7 \mathrm{~g} / \mathrm{cm}^{3}\right)$ aluminum, however, indicate that the Lee and More model predicts the real and imaginary parts of optical conductivity to within $20 \%$. Only at the lower temperature $(<0.1 \mathrm{eV})$ does the Lee and More model seem to over estimate the imaginary part of the conductivity and fall outside of our error bars.

We also examined the temperature dependence of the conductivity using this same technique using 170 and $230 \mathrm{~nm}$ aluminum foils heated with the intensities of $(2-4) \times 10^{14} \mathrm{~W} / \mathrm{cm}^{2}$. We find that in all cases we drive the same conductivity though the arrival of the heat wave and subsequent shock waves varies with the choice of intensity and target thickness. This consistency in our data gives us good confidence in the validity of our technique for deriving conductivity as a function of temperature.

In conclusion, we have presented experimental studies of electron ac conductivity in warm dense aluminum. Using single-shot frequency-domain interferometry with chirped pulses, we have conducted measurements of the dielectric constant of dense aluminum in the $0.1-1.5 \mathrm{eV}$ range. Calculations of conductivity based on the Lee and More conductivity model are in reasonable agreement with our measurement. Both free- and bound-electron contributions to the optical conductivity were taken into account in our calculation. The imaginary part of the optical conductivity exhibits Ohmic-like conductivity in the studied temperature range. We find that the Lee and More model predicts the real and imaginary parts of the conductivity within $20 \%$ and is particularly accurate at temperatures $>0.5 \mathrm{eV}$.

This work was supported by the DOE National Nuclear Security Administration under Cooperative Agreement No. DE-FC52-03NA00156.
[1] H. M. Milchberg, R. R. Freeman, S. C. Davey, and R. M. More, Phys. Rev. Lett. 61, 2364 (1988).

[2] A. Ng, P. Celliers, A. Forsman, R. M. More, Y. T. Lee, F. Perrot, M. W. C. Dharma-wardana, and G. A. Rinker, Phys. Rev. Lett. 72, 3351 (1994).

[3] K. Widmann, T. Ao, M. E. Foord, D. F. Price, A. D. Ellis, P. T. Springer, and A. Ng, Phys. Rev. Lett. 92, 125002 (2004).

[4] Y. Ping, D. Hanson, I. Koslow, T. Ogitsu, D. Prendergast, E. Schwegler, G. Collins, and A. Ng, Phys. Rev. Lett. 96, 255003 (2006).

[5] A. Ng, A. Forsman, and G. Chiu, Phys. Rev. Lett. 81, 2914 (1998).

[6] E. Tokunaga, A. Terasaki, and S. Kobayashi, Opt. Lett. 17, 1131 (1992).

[7] Y. T. Lee and R. M. More, Phys. Fluids 27, 1273 (1984).

[8] F. Brunel, Phys. Rev. Lett. 59, 52 (1987).

[9] M. Takeda and S. Kobayashi, J. Opt. Soc. Am. 72, 156 (1982).
[10] K. Y. Kim, I. Alexeev, and H. M. Milchberg, Appl. Phys. Lett. 81, 4124 (2002).

[11] R. Evans et al., Phys. Rev. Lett. 77, 3359 (1996).

[12] J. T. Larsen and S. M. Lane, J. Quant. Spectrosc. Radiat. Transf. 51, 179 (1994).

[13] M. Born and E. Wolf, Principles of Optics (Pergamon Press, Oxford, 1980), p. 55.

[14] N. W. Ashcroft and K. Sturm, Phys. Rev. B 3, 1898 (1971).

[15] D. Fisher, M. Fraenkel, Z. Henis, E. Moshe, and S. Eliezer, Phys. Rev. E 65, 016409 (2002).

[16] A. N. Mostovych and Y. Chan, Phys. Rev. Lett. 79, 5094 (1997).

[17] A. G. Mathewson and H. P. Myers, J. Phys. F: Met. Phys. 2, 403 (1972).

[18] K. Y. Kim, B. Yellampalle, J. H. Glownia, A. J. Taylor, and G. Rodriguez, Phys. Rev. Lett. 100, 135002 (2008). 